Meta

Journal des traducteurs

Translators' Journal

\title{
La circulation terminologique et les rapports entre science, technique et production
}

\section{Louis Guespin}

Volume 40, numéro 2, juin 1995

Usages sociaux des termes : théories et terrains

URI : https://id.erudit.org/iderudit/002980ar

DOI : https://doi.org/10.7202/002980ar

Aller au sommaire du numéro

Éditeur(s)

Les Presses de l'Université de Montréal

ISSN

0026-0452 (imprimé)

1492-1421 (numérique)

Découvrir la revue

Citer cet article

Guespin, L. (1995). La circulation terminologique et les rapports entre science, technique et production. Meta, 40(2), 206-215. https://doi.org/10.7202/002980ar
Résumé de l'article

Le classement des termes scientifiques et techniques en domaines a pu convenir en des périodes plus statiques. La généralisation des problématiques pluridisciplinaires met sérieusement en cause cette vision commode. Mais les nouveaus rapports entre science, technique et production compromettent plus encore celte répartition: la hiérarchie traditionnelle du " savant » à I'« ingénieur » puis à I'« entrepreneur » est compromise tant dans l'imagerie populaire que dans la pratique sociale: qui est désormais à l'initiative dans tel projet mettant en oeuvre à la fois des savoirs, des savoir-faire et un dispositif de production? Pour être opératoire de nos jours, la terminologie devra tenir compte de la réalité du transfert d'information, et se donner pour lâche non de prescrire le bien-dire dans ce domaine, mais de faciliter les échanges dans une sphère d'activité. 


\title{
LA CIRCULATION TERMINOLOGIQUE ET LES RAPPORTS ENTRE SCIENCE, TECHNIQUE ET PRODUCTION
}

\author{
LOUIS GUESPIN ${ }^{+}$ \\ limuersité de Rouen. Rouen. France
}

\begin{abstract}
Résumé

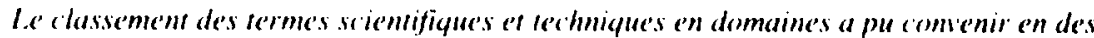

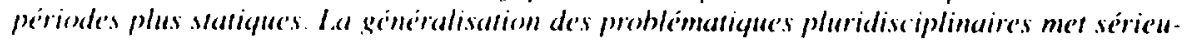

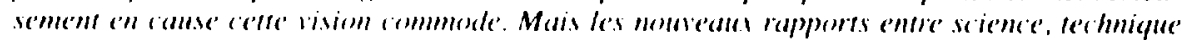

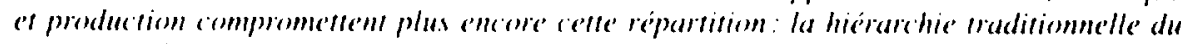

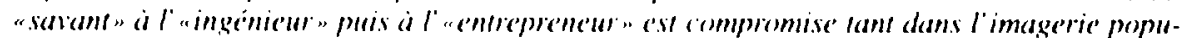

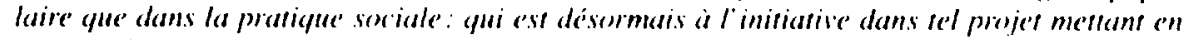

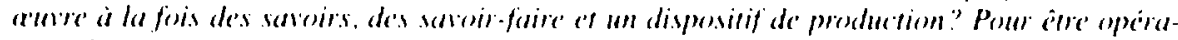

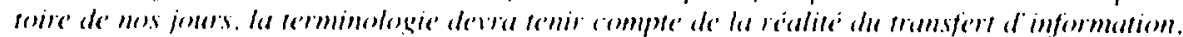

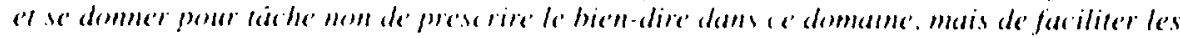

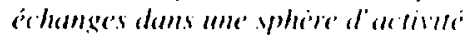

Réfléchissant sur les langues de spécialité, Allal Assal s'amuse, dans sa thèse (Assal 1992). à suivre les chemins tracés par divers chercheurs, qui se plaisent à dénombrer les disciplines reconnues ici ou là : 1. Dahlberg, par exemple, a recensé 730 désignations de spécialités scientifiques, incluant $6.5(k)$ sous-spécialités réparties en 9 groupes thématiques tels que l'économic. la biologie. elc. (1986). Pour un pamphlet contre le positivisme et la manie des démarcations, cette liste peut être utile pour l approche des problèmes terminologiques qui se posent à la circulation de l'information scientifique et technique. l'intérêt est moins évident. sauf à voir là une opportunité pour réfuter la vision classique des "langues de spécialité" (que nous abrégerons désormais en LSP): y en aura-t-il 6 50)" ou 39'? el que se passe-t-il quand trois aspécialistes» de trois de ces uspécialités" entrent en interaction verbale: que parlent-ils? un méta-1.SP? un nouveau L.SP? Tout ceci est un peu farfelu.

Dès qu'on entre dans la problématique d'un L.SP strictement assigné à un domaine, rien ne tient: sans cesse la pratique. l'évidence de la réalité langagière, viennent démentir les postulats les plus indispensables à l'attitude descriptiviste fondée sur les "domaines». qui caractérise la terminologie classique.

Par exemple. I'histoire, que la théorie d'Eugen Wüster chasse par la porte, revient obstinément frapperr au carreau. si fort même qu elle ébranle la belle construction: comment expliquer sans l'histoire les constantes survivances lexicales. en terminologic comme dans le langage quotidien? Quand on constate que coxistemt en biologic germe, microhe et michermganisme, peut-on simplement ignorer deux des trois termes? ou voir une différence sémantique là où il n'y en a pas? (On peut dire: «il faut dire"; peut-on honnêtement dire: "on dit seulement»"? Bien sûr. ge"me est vieilli, mais des livres el des cours de biologie l'utilisent encore, et il est moins désuet en biologie médicale (voir portér de ge'me') yu én microbiologic: miroble fait un peu pastorien, fin de siecle, mais il joue encore un rôle important, ne serait-ce que par ses dérivations (généfique microbiemmé, substance microbicidel: enfin. micro-onganisme n'est pats la panacée: si son signifiant semble limiter le

Mr'tu. XI.. 2. 1995 
sens au "tout petit", à ce qui n'est visible qu'au microscope, le concept microbiologique comporte dans son extension algues et champignons, pas toujours si microscopiques que ça... Et l'on ne peut s'en tirer en assignant aux trois signifiants, pour mettre un peu d'ordre. des contenus conceptuels clairement distincts: ce ne serait pas vrai. Il faut bien accepter une possibilité de synonymie. due certes à la délimitation incertaine du concept pour chacun des trois signifiants, mais plus encore à des dates d'apparition différentes. c'est-à-dire à l'éclosion des trois termes dans des systèmes scientifiques et technologiques à la fois partiellement différents mais aussi partiellement héritiers les unns des autres. On ne voit pas qu'on puisse ici échapper à l'histoire.

Il en irait de même si l'on s'intéressait à la dénomination des enzymes. Au XIXe siècle, on a découvert les diastases : c'est encore ainsi que les livres de "sciences nat" de ma jeunesse les désignaient. La suffixation en -ase de ces diverses «diastases» (amvlase. etc.) a entraîné chez les anglo-américains la tentation de les baptiser «ases», par troncation: on connaît le procédé, qui donne aussi bien teen en langage quotidien (to be in one's teens. te(")-ager) que etic's et emic's en linguistique (par troncation à droite de phonetic s/phonemics. morphematics/morphemic's). Il se trouve que c'est c'nzyme' qui l'a emporté, parmi les trois candidats au poste d' "archilexème". c'est-à-dire de concept englobant toutes les substances en -ase. Ici encore, va-t-on nier la possibilité de concurrences et de conflits en synchronie?

À l'exact opposé, fermentation. aujourd hui, ce sont au moins trois notions, dans le même "domaine" des biotechnologies: c'est le phénomène hiochimique exploité par les techniques, empiriques pendanı des millénaires, mais de plus en plus dépendantes de la science. utilisées pour la fabrication du vin. de la bière, du pain, ou la conservation des produits lactés: mais c'est aussi le phénomène microbiologique étudié par Pasteur, défini et délimité par lui comme ala vie sans airm. c'est-à-dire la fermentation anaérohie: et enfin. à l'heure de la bio-industrie. c'est aussi. et ceci sans qu'il soit besoin de le préciser dans une revue de biotechnologie comme Biofiutur, "tout ce qui se passe dans un fermenteur". c'est-à-dire diverses réactions, dont des fermentations aérobies et non aérobies; cette définition, que je forge de façon perverse mais en connaissance de cause, sera d'ailleurs parfaitement circulaire, puisque le fermentetr sera non moins valablement défini comme "l'appareil dans lequel a lieu une fermentation". bien sûr au sens industriel défini dans la phrase précédente.

Ces quelques exemples sont prélevés à nos recherches de groupe: Véronique Pierzo, en particulier, a étudié le vocabulaire du génie de la fermentation (1991), et Allal Assal (1992) s"affronte à la question des "domaines" et des "LSP». Il va de soi qu'on pourrait multiplier les exemples de cas où soit plusieurs signifiants se concurrencent dans le lexique d'une "spécialité". sans autre différence claire que le sentiment de désuétude ou de modermité, et les cas où un même signifiant peut. à l'intérieur d'une même sphère d'activité, recouvrir des pratiques techniques ou des concepts scientifiques très différents. On ne fera pas l'économie de l'histoire. histoire des sciences, des techniques et des formes de la production.

Mais la synchronie elle-même a ses pièges. Acceptons les 6500 spécialités du collectionneur de disciplines cité plus haut, prévoyons-en généreusement $700(0)$ pour dans six mois: l'essentiel reste que l'étude des textes que le spécialiste adresse aux spécialistes est peut-être la moins intéressante qui soit: se polariser sur eux. c'est un peu comme fonder une étude de l'homme sur la tératologie. Il est centes à peu près vrai que la logique discursive d'une langue de spécialité. duns l'écrit scientifique officiel au niveau de la communication de congrès ou de l'arricle de revise spécialisée. tend a une assez forte spécificité: l'univocité des termes y est recherchée. le recours à un symbolisme propre n'a pas à y être explicité, phraséologie et rhétorique peuvent y être imposées. Mais ces textes ne sont nullement premiers: l'attitude qui consiste à donner primat à leur étude correspond 
à celle des anciens grammairiens qui cherchaient à décrire "le français» à partir de grands textes littéraires: on excipe d'une langue en rupture a la norme', à la logique du langage. pour dire la norme: les textes scientifiques "purs" offrent eux aussi nombre de décalages par rapport à la mise en circulation courante de l'information scientifique. D'abord, par le système linguistique même auquel il est fait recours: de nos jours, bien des sciences se pratiquent en français mais rapportent leurs résultats en anglais: dans le cas de ces discjplines, le texte scientifique "pur" en français est ralre, el son artificialité augmente d autant: en biologie par exemple, la publication scientifique de haut niveau en français est, on doit le déplorer, insignifiante: la prendre pour centre de létude terminologique, ce serait non seulement privilégier un niveau sophistiqué de communication, mais encore passer à côté de la vérité de l'interaction scientifique de niveau international dans la discipline, puisque celle-ci se fait en anglais.

Même abstraction faite de celte considérable ombre au tableau, il y a encore bien des faits qui vont contre le primat donné à la ulangue de spécialité"; par exemple. le fait que tout commence par loral. un "oral de la science", selon la formule de François Gaudin, dont à peu près personne tie parle. Or le laboratoire a son jargon. et celui-ci est souvent bien éloigné de la langue lissé el convenue des congrès et articles. Le même objet y est désigné par tout un paradigme de signifiants, du formel au familier. Et aussi, loin de cultiver l'univocité, on joue sur les mots, au labo comme ailleurs. Une anecdote: une chercheuse, célibataire un peu prolongée. sort de son labo) en clamant «j ai trouvé le mâle»; il s"agit bien de "communicition entre spécialistes", mais on se doute que le sens de "mâle" en langue quotidienne a été malicieusement convequé par les auditeurs!

Entin. si le discours scientitique upur" pouvait être isolé, s'il se révélait univoque, lavé de toute ambiguïté. de toute synonymie. de toute homonymie. on pourrait certes l'étudier, pour le plaisir de démonter un si bel objet: mais le terminologue n'aurait guère que des constats à faire: celle langue si parfaite, angélique ou divine, $n$ aurait guère besoin du recours des hommes. Or on voit vite qu une forte contradiction existe entre cette idéalisation du discours scientifique el les réalités de la pratique: le discours de la science. qu' elle soit en train de se faire ou de se communiquer, a besoin de régulations constantes, d'un bricolage interactif' au cours même de l'interaction. L'autoréglage par le consensus des «savatnts" n'est qu'utopie: Evelyne Bulot (1988) a montré, pour divers types de discours, yue le dystonctionnement est élément constitutif de l'interaction: l'interaction langagière consiste en un constant réglage, toujours imparfait, toujours à négocier: l'étude des textes $n$ indique pas que. pour quelque niveau que ee soit, le discours scientifique échappe à cette règle communc.

Je n'énumérerai pas ici toutes les évidences, je veux juste indiquer le besoin où nous sommes d'échapper à une vision abstraite et platonicienne du discours scientifique. Je me contenterai donc d un dernier argument. fondamental à lui seul. et sur lequel nous reviendrons plus loin: la nécessité de prendre en compte les modes et les canaux réels de la communication scientifique et technique.

D'abord, remarquons que je viens de dire «scientifique et technique», après avoir raisonné sur le discours scientifique. Que s'est-il passé"? Un glissement commole et banal: le terminologue, quand il fait un peu de théoric, raisonne sur la pointe extrême du discours scientifique; cest là qu'il peut trouver cette pureté qu'il assigne alors au LSP. Mais le sigle LSP est trop commode; selon les aluteurs et les besoins, il va signifier language for scientific purposes ou language for speecific parposeses. Le résultat de cette incertitude est que ce qui est dit du discours scientifíque va être considéré comme établi pour tout langage de spécialitc. Le français lui aussi lend un piège, avec son syntagme assonancé "scientifique el technique"; (ce syntagme, qui tend au figement dans des expressions où la langue n'est pas en cause (par exemple, la revolution sciemifique et techmique'). est abusivement 
transféré à la réflexion métalinguistique. dans des formules commme «le discours scientifique et technique”. “les vocabulaires scientifiques et techniques".

Or, si la langue d'une spécialité scientifígue peut componter une pointe ultime de fonctionnement presque entièrement volontariste et codé par convention, il n'en est rien à coup sûr de la langue d'aucune technologie. Et ceci, par nature: la technologie applique la science, elle n'est donc jamais elle-même sans le discours d'une ou de plusieurs sciences. Elle est le fruit de la rencontre du "scientifique" et de l'ingénieur: il faut bien que le dialogue s'établisse, dialogue où l'un apporte sa discipline et l'autre sa connaissance des matériels et méthodes, les sciencés de l'ingénicur.

Nous voici dans l'engrenage fatal: si nous suivions les terminologues tenants des LSP, nous ne serions plus terminologues, puisque nous nous préoccupons d un dialogue non plus interne, mais par-dessus les harrières des spécialités et des domaines. El nous découvrons alors que la science. comme la technologie, est essemtiellement faite de ces interactions impures. Le "savant" parle à l’ingénieur: mais ne parle-t-il pas aussi à des étudiants? à des edécideurs»? à ceux qui peuvent lui procurer des crédits avec leurs appels d'offres, ou à ceux qui rémunèrent son labo par des contrats'? à des juristes, enfin. quand il s'agit d’établir propriété intellectuelle et commerciale dans le cadre d'un dépôt de brevet?

Que le scientifique s'adresse de temps en temps à un plus large public que celui de ses pairs, le descripteur en tient parfois compte, et l'on a prévu à cet effet le concept de rulgarisation. Mais cette opposition entre discours scientifique et discours de vulgarisation présente le défaut de donner bonne conscience au descripteur du discours pur: il y aura pur et impur, scientifique et vulgarisé.

J'espere avoir fait sentir qu' en fait il s'agit toujours d'un continuum: tout simplement. comment définir l'interaction quand deux ou trois scientifiques "purs" ont à côté d'eux un stagiaire ćtudiant, dans le même labo? ou quand ces mêmes "purs" présentent leur projet au CNRS ou à un ministère'? Jusqu'ici, mon exposé $n$ 'a visé qu un seul but: déstabiliser. On reconstruira plus loin. Les adomaines». ça ne va pas: la langue de spécialité. ce n'est pas un concept. La formation discursive "communication de congrès", réalité indéniable et intéressante. ne saurait permeltre d'éludier la logique des interactions où s'élaborent et se transmettent science et technique contemporaines.

$\mathrm{Ai}$-je fait autre chose que d'indiquer, en creux. la nécessité de recourir à la sociolinguistique? On sait que cette discipline prend le relais d'une linguistique qui a achoppé sur son opposition entre langue et parole. Cette dichotomie fondatrice, qui a permis la naissance d'une linguistique du système. a échoué à intégrer l'aspect social du langage: elle empêchait toute vision en continuum. La sociolinguistique va donc s"intéresser à nombre de paramètres sociaux nécessaires à l'établissement de la causalité en matière de variation langagière. Elle va s'interroger sur qui parle, avec qui, en quel lieu. On aura alors les travaux d'un Basil Bernstein ou d'un William Labov. Malgré l'évident intérêt de ces recherches, on peut, a posteriori. leur reprocher de négliger certaines variables: or diverses theories ou diverses approches sont venues enrichir ces travaux pionniers. Aux questions énoncées plus haut, on peut par exemple adjoindre "pour quoi '" ". Modéliser la réponse à cette question. c'est l'affaire de la pragmatique, née hors de la sociolinguistique. puisque ce sont des considérations logiques qui ont présidé à sa naissance. mais nécessairement entraînée vers le social: qui donne au langage son pouvoir d'agir sur le monde. sa dimension d'action. sinon la société, les groupes sociaux, les structures de la sociabilité? Voici donc la sociolinguistique soucieuse de prendre en compte les acres de langage: ce que l'on fait, en tant qu'acte social, quand on parle. En France, une autre approche de la dimension sociale du langage a aussi fait progresser la connaissance des structures langagières: l'analyse du discours, née des techniques de l'américain Zellig Harris et 
fécondée. sous l'impulsion de Jean Dubois, par la problématique historique et sociale de la linguistique française.

On peut alors légitimement parler de sociolinguistique, et, pour la France, selon la formule de Jean-Baptiste Marcellesi et Bernard Gardin, de linguistique sociale (1974). Le point de vue de mon groupe de recherche est que l'on peut el doit s'appuyer sur cette discipline mûre pour dépasser les apories d'une terminologie classique qui montre ses limites. II est. à nos yeux. lemps de reconnaitre l'existence d'un nouveau courant en terminologie, un complexe d'attitudes pratiquess è d'élaboration théorique yue nous appetons sociotominologic'.

La socioterminologie s'appuie sur les concepts et démarches de la sociolinguistique: si Louis Guilbert ne nous avait pas été trop tôt enlevé. on peut penser qué son souci de marier lexicologie du systeme et prise en compte de l'histoire et du discours aurait fait naitre plus lôt la nouvelle discipline: on peut voir dans La crécatrité levicale (1975) un ouvrage précurseur de la socioterminologie.

Si la socioterminologie combal la polarisation sur le discours "pur", sur l'univocité, etc., elle sait reconnaître, dans tout usage scientifique et dans tout usage technique de la parole. des paramètres spécifiques, régulateurs plus ou moins dominants de l'interaction verbale. La tendance à l'univecité reste la règle, même si elle ne réalise jamais son idéal: les agences de normalisation y sont plus efficaces qu ailleurs, même si elles sont, là aussi. fréquemment bafouées: les formes de l'interaction discursive y sont plus codées què en LGP (kanguage for general purposes). mème si elles peuvent toujours être tournées. Enfin. la concurrence de langlais international, qui constitue parfois la seule langue admise pour la communication écrite internationale, y est plus forte qu ailleurs.

Le vrai problème de la socioterminologie n'est donc pas la négation de ce qui a éte abusivement porté a l'absolu, mais la prise en compte des divers facteurs en action, et de leffet qu'ils ont.

\section{I.E RÖL.F SOCIAI. AT TIEI. DE. I.A TERMINOL,ORIF:}

La terminologie naît, légitimement, dans l'empirisme. Dans les années 30 de notre siècle, divers facteurs se sont conjugués pour réclamer l'intervention de chercheurs dans le domaine du terme. Évidemment, les premiers soucis sont de standardisation: le corur de cette discipline naissante bat à Vienne, où travaille Eugen Wüster. le "père de la terminologie»; d'où la création dans celte ville de I'ISA. ensuite ISO (International Standardisation Organisation); de nos jours, avec ISO et Infoterm. I'Autriche reste le centre de cette terminologie "classique". De leur cote. les Soviétiques ont eu à faire face vers le même temps aux problèmes posés par le caractère multinational de l'URSS: même si la politique des nationalités de Staline est brutale et superficielle, comme on s'en aperçoit aujourd hui. les engagements pris dans la perspective du respect des cultures et langues locales entrainent un énorme effort d'éutipement linguistique de langues souvent exclusivement orales. et parlées parfois par quelques milliers de locuteurs seulement. Cet interventionnisme linguistique réclame une théorie terminologique: les Soviétiques acceptent alors grosso modo les positions de Vienne, malgré une kendance plus forte à la prise en compte des facteurs sociaux et de la réalité du langage.

Toutefois, dans lensemble, la théorie de ces terminologies "classiques" est mince. et repose sur des postulats contestables: le terme scientifique et technique se distinguerait clairement du mot du langage quotidien par son univocité: le disconers scientifique et technique se caractériserait par son absence d'ambiguité: le terminologue aurait à traiter de drmaines dont les notions ou concepts seraient indifférents à la langue qui les exprime: son rôle serail alors de décrire la structure du résecus des motions dans un domane 
dét'erminé. l'enregistrement des formes qui correspondent à ces notions faisant ensuite l'objet d'un nettoyage. d'une rationalisation, par suppression des doublets (synonymes). et création. par des commissions de spécialistes réunis ad hoc. des néologismes nécessaires. À cette évocation, qui $n$ 'ést guère caricaturale, on comprend comment l'image d'un discours scientifique fonctionnant sans défaillance a pu naître: le projet de standardisation a été confondu avec la tâche de description. l'objet idéal visé a amené à sublimer le réel observé.

Cetle optique est à nos yeux désuète, parce qu'elle repose sur les postulats que nous avons énumérés, et qui ne sont pas tenables après cinquante ans de progrès en recherche linguistique. À la vérité. cette terminologie ignore pratiquement l'ceuvre de Saussure. même quand elle s'en réclame: la condamnation de la vision de la langue comme une nomenclarure où les «mots». c'est-à-dire les formes écrites ou prononcées, correspondraient à des "concepts», qui relèveraient de l'élude psychologique. est une base nécessaire pour Saussure: or la théorie de Wüster et la pratique terminologique "classique" reposent sur la postulation de ce rapport direct entre les “lermes». simples formes écrites, el les «notions». internationales et propres à la discipline scientifique. sans intluence de la langue.

Ce sont donc les hases mémes de la linguistique moderne qui manquent à la terminologie ainsi conçue. Mais cette discipline $n$ 'a pas même pris le train en marche: elle aurait pu rejoindre l'aventure linguistique par le biais de l'analyse du discours, ou de la pragmatique en tant qu étude du rôle du langage dans l'action humaine, ou par celui de la sociolinguistique.

\section{MODELLISER L.F CIRCUIT RÉFL DU TRANSFERT D'INFORMATION}

La terminologic a manqué les rendez-vous que lui proposait la linguistique. La discipline a toutefois évolué, essentiellement sous la pression de la pratique. Le cas le plus net est celui du Québec, où la politique volontariste en matière de sauvegarde du français par son équipement terminologique à amené à poser en vraie grandeur el de façon globale les problèmes de l'adéquation de l'équipement terminologique à la demande' sociale, ainsi que de l'aide que peut apporter la terminologie à l'erymession de ces besoins.

Je me contenterai de cet exemple d'un cas où lat pratique entraine une réflexion théorique nouvelle. peu éloignée de ce que notre groupe entend par socioterminologie.

Ceci dit. il nous faut bien prendre conscience que les besoins terminologiques changent. à I'heure actuelle. en fonction de deux paramètres au moins. Le premier n'est pas nouveau: c'est le fait même' de la révolution scientifique et technique. et de son accélération. avec ses exigences dénominatives $(5(X) 000)$ nouveaux composés chimiques "sortent" tous les ans), avec ses besoins de régulation internationale de l'échange d'information. etc. Je n'en parlerai pas ici. Le second est rarement pris en compte dans la réflexion des terminologues. C'est la mutation des rapports entre science. technique et production. Les études d'anthropologie. de sociologie du travail, d'ergonomie, etc., qui enregistrent cette mutation et tentent de l'analyser et de la caractériser. n'irriguent guère la pratique terminologique. alors que leur prise en compte est totalement nécessaire si nous voulons construire une terminologie en prise sur le réel.

On ne peut se contenter d'une terminologie décrivant ou codifiant (de manière d'ailleurs assez fantasmatique, car enfin, qui suit les recommandations des ministères ou de l'AFNOR en matière de comportement lexical dans les sciences et les techniques?) les pratiques langagières au plus haut niveau de la science, vocabulaires de congrès internationaux, de revues scientifiques internationales, standardisation par d'éminents spécialistes de la discipline. Il est indispensable de replacer la problématique de la terminologie dans le circuit réel de la production et du transfert de la connaissance scientifique et 
technique. Et ce circuit n’a ni la "simplicité" ni la "pureté» à laquelle en sont restés les terminologues traditionnels, mème quand leur matériel se sophistique avec l'informatique. les banques de données. les systèmes experts.

L.adéquation au réel ne viendra pas de la seule sollicitation des techniques nouvelles de l'information: il faut d'abord rechercher la bonne compréhension. la bonne analyse et

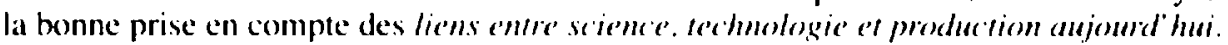

La vision qu'on a de la science et de la technique porte. quoi qu'on en ait. la marque de $25(x)$ ans de logese et de techen'. Le logos, cest a la fois le verbe, qui se fait chair dans la spéculation mystique, mais qui. dès avant, dès Platon. désigne les idées.se seule réalité dont les objets du monde ne sont que des reflets pâles et déformés. et c'est en même temps la science, toutes les sciences; la techné. cest l'artisanat. quelque chose d'assey proche de la servitude, quelque chose de vulgaire; au mieux. cest l'idée alourdie par lépaisseur de la chair des choses concrètes. "Saintes douceurs du ciel. adorables ldées". dira encore Polyeucte au XVII" siècle. Au XVIII", c'est peut-être par l'Finchlopédie que la Révolution s'annonce le plus clairement. dans cette machine de guerre idéologique qui ose faire leur place aux lechniques et aux carts méchaniques".

Dans cet immense laps de temps, plus de $2(K)$ ans, la science parait presque entiorement détachée de la lechnique, ainsi d’ailleurs que de l'utilité sociale. Je dis "paraît», car il sagit sans doute plus d'un mode idéologique de répartition des pouvoirs symboliques et des róles sociaux que d'une réalité pratique. mais ceci serait l'objet d'une autre ćtude. Pendant $2(k)$ ans. en tout cass, simpose celle vision d une coupure entre la science et les techniques: la science, occupée d'idées pures. chargée de décronrir. sous la face aléatoire des choses. le réel du monde, et non de sen approcher en une approximation interminable. comme on le sait aujourd hui: en face de la science. les techniques. simples. pratiques. empiriques. qui ne méritent ni réflexion ni explication, mais reconduction. transmission de savoir-faire, au mieux amélioration par coup de chance, observation ou habileté. On oppose volontiers les "arts libéraux", kes disciplines scientifiques, théologie comme mathématiques, dignes des hemmes libres. el les "arts méchaniques", réservés aux "gens méchaniques", plus ou moins serfs ou bourgeois. Si le monde concret n'est que le reflet ou le sous-produit du monde des idées, comme le prone lidéalisme, de Platon à Hegel, pendant plus de deux millénaires, ators la science est du côté des idées, et la technique du côté de la matiere vile.

Bien sûr. si cést là la partition dominante dans les têtes pendant ce long laps de temps. il y a des esprits marginaux pour refuser le dogme. et l'évolution des conditions économiques et politiques entraine une évolution du rapport entre science et technique.

Du premier point - les précurseurs, les déviants - nous ne dirons rien ici, malgré la fascination que procure la complétude intellectuelle d'un Léonard de Vinci, artiste génial, mais aussi scientifique el ingénieur. Plus exactement. évorquer la transformation des rapports entre science et technique, cela peut se faire de deux façons: en balisant te terrain de noms d'lommes qui ont su lier plus tôt que d'autres. et souvent malgré les autres, science el application: Léonard déji cité, mais aussi Pascal, Descantes et tant d'autres: lautre manière, moins événementielle. consistera à se demander à quelle évolution des rapports correspond ce paradigme de novateurs.

On peut dire que les "temps modernes", héritiers des grandes innovations techniques rendues possibles au Moyen Âge par les progrès d'une concentration urbaine sans esclatvage. d'une part précipitent ce mouvement de novation technique, et d'autre part voient poindre une attitude de réllexion sur les techniques, une curiosité pour les arts mécaniques. C"est un processus lent. à cause sans doute de la pesanteur du vieux mépris pour la technique hérité de l'Antiquité et évoqué ci-dessus; mépris qui n'est pas encore mort dans toutes les consciences aujourd hui : ne réserve-t-on pas. en France, l'enseignement 
technique aux "moins doués"? Mais le principe est posé: la science peut faire la théorie de la technique. et. accessoirement. améliorer celle-ci. Cet objectif d'optimisation est sûrement secondaire, au moins dans les consciences: les lectures latines font certes méditer les jeunes gens sur Pythagore. mathématicien et en même temps ingénieux inventeur de machines de guerre optiques; mats s'agit-il dencourager un rapport entre science et application à la vie matérielle, ou d'illustrer la force d'esprit de l" "homme illustre" capable de se distraire de la spéculation pure par l'amusante invention d'un gadget pour militaires?

Nous avons besoin de ce détour pour dire pourquoi la terminologie doit. pour chaque époque, tenir compte de l'état des rapports entre science, technique et production: signalons qu'au stade évoyué, celui des temps modernes, en gros donc, du XVIe au $X I X^{c}$ siècle. le vocabulaire technique ést exclu de toute description lettréc, au même titre que les mots "bas" ou «populaires". Je l’ai rappelé plus haut. l'introduction de considérations sur les "arts et les techniques" dans l'Fincliclopédic de Diderot et de d'Alembert est une véritable novation: sans doute en position quelyue peu volontariste, à la pointe extrême d'une évolution des mentalités en train de se faire, elle porte témoignage de cette possibilité nouvelle de réfuter la croyance à l'indignité des techniques.

I ce XIXe siècle est un siècle charnière. La machine à vapeur signe la première révo. lution scientifique et technique: révolution technique, puisque la mâtrise de l'énergie transforme fantastiquement le rendement des investissements industriels, mais révolution screntifique parce que la découverte de l'unilé des formes d'énergie, complémentairement a la réfutation du "phlogistique" et à la naissance de la chimie avec Lavoisier, vient répondre à dess questions insolubles dans le paradigme mécaniste du XVIII" siècle.

La liaison sciencé-technique tend dès lors à être conçue comme le mode d'être de la scrence: quand la technique s améliore, alors la science peut progresser; et. en retour, la scence en progrès pourra optimiser la technique.

Certes, les choses ne sont pas si nettes dans les esprits, en particulier en matière de dignité des fonctions sociales: on a une Académie des Sciences, et non des Techniques: on a des facultés des Sciences cent cimquante ans avant d'avoir des universités technologiques. C'ertes, le Consulat créc Polytechnique, mais il s'en faut de cent ans pour que le polytechnicien rejoigne, dans le Panthéon de l'imaginaire social. le normalien lettré: et on sall que Polylechnique a écé délourné de sa vocation: s'ils sont définissables, les $X$ sont plutôt des scientifiques que des lechniciens.

Liessentiel est que, à côté de sa fonction de comnaissance théorique du monde, la science est désormais chargée d'une fonction d'amélioration de la technique. Et c'est pour désigner ce nouveau mode de coexistence. celte mise en rapport systématique de la science et de la technique, yu’a été créé le terme de tochmologic'.

Toutefois, d'un point de vue philosophique, on peut penser que ces deux fonctions $n$ 'en font qu une: on ne connait qu én transformant. et donc. la connaissance scientifique du monde entièrement humanisé, socialisé, où nous vivons, ne peut progresser sans Iransformation constante de nos moyens d'agir sur lui par les techniques.

D'un point de vue pratique, les structures institutionnelles, enseignement. profils des carrières. spécialités. reconduisent cependant un cloisonnement: moins hiérarchique que par le passé. il est toutefois socialement reconduit: on est chercheur ou ingénieur: si l'on considere par exemple la terminologie en vigueur au CNRS. une hiérarchie des carrières existe entre cherh h'lor (générique pour directeur de recherche. maître de recherche.

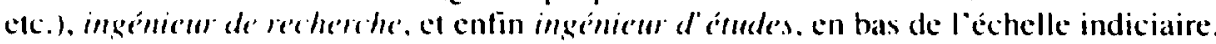

Toutefois, le rapprochement est indéniable: on reconnaît. entre recherche et production. des zones intermédiaires dont l'appellation même pose problème: reche'che' appliquéce par opposition à recherehe fondame'male, recherehe-développerment. elc. Dans 
cette zone. plus ou moins vaste et plus ou moins tranchée selon les disciplines et les sphères d'activité, coopèrent sciences fondamentales, souvent convoquées de façon pluridisciplinaire. et sciences de l'ingénieur: il s'agit d'anticiper sur la future production. en testant les nouveautés du laboratoire de recherche fondamentale sans engager les frais d'une chaîne de production au rendement linancier encore hypothétique: des phénomènes se produisent en effet au laboratoire. par exemple en fermentation, qui seront difficiles a reproduire dans l'industrie, car le changement d'échelle peut entraîner des problèmes inattendus (homogénéisation, ventilation, refroidissement...): le «stade du pilote" permet de tester un niveau intermédiaire, cette interface engageant généralement. du côté des chercheurs comme de celui des ingénieurs, des spécialistes de recherche appliquée, qui. outre celle interaction, se chargent du dialogue avec la recherche fondamentale (demande d'amélioration. de complément de recherche en laboratoire) el avec l'industrie (marges tolérées. surcoûts envisagés. faisabilité "en grand").

Dans ce rapprochement pratique, les barrières tendent à s'abaisser: on verra l'ingénieur poser ses problèmes scientifiques, et le chercheur anticiper sur les contraintes des stades appliqués: il y a dès lors un monde commun. chacun anticipant les problèmes du lieu d'origine de l'autre. Il va de soi que les implications terminologiques sont immenses: ce dialogue n'est ni celui de l'information scientifique spécialisée. ni celui de la communication dans l'entreprise.

Mais il y a plus nouveau encore: la notion même de pilotage par l'atal. Celte formule a été combattue. à cause de son caractère utilitariste, qui retourne maladroitement le sens historique de la courroie de transmission entre science et technique, en prévoyant, en lieu de dialogue et de coopération. un statut minorisé du chercheur. Et contradictoirement, deuxième défaut de la formule, on peut penser que la métaphore même d'un amomt el d'un aral n est pas sans reconduire une vision inutilement hiérarchique: dans un dialogue, y at-il nécessairement relation de domination? Non seulement les connaissances se complètent, mais on peut mème dire qu elles n existent que d'être partagées: aussi compétents soient-ils dans l'absolu, le chercheur et l'ingénieur $n$ 'auront rien à se dire $s^{\prime} i l s n^{\circ}$ ont un fonds commun, sur lequel leurs originalités respectives peuvent fructifier.

Pilotage par l'aval: expression absurde donc, mais notation d'un fait nouveau: la commande sociale à la science. qui peut émaner de divers lieux d'activité. formulée par différentes instances porte-parole. Pour la recherche sur le cancer ou le Sida. c'est la communauté entière qui est intéressée. et ce sont des instances précises qui demandent: instances traditionnelles, comme le ministère de la Santé, ou ad hoc, comme la Ligue contre le cancer. En biotechnologie, la demande peut venir d'une branche industrielle ou d'une entreprise particulière: et cette demande peut avoir pour origine divers services d'une entreprise: l'ingénieur de production peut vouloir améliorer un rendement, mais le spécialiste du marketing peut avoir remarqué un "trou» dans se panoplie d'organismes recombinants, ou de processus, et conclure après élude du marché qu'il y aurait une demande pour un procédé. un organisme ou un gène à imeenter.

Cette évocation des rapports nouveaux entre science, technique et production. nécessairement brève et impressionniste. permet je l'espère de comprendre combien lit notion de discours "pur", vraisemblablement impropre pour toute époyue. doit être plus encore relativisée de nos jours. Le "savant" comme le "lechnicien" sont désormais plus souvent pris dans des structures de dialogue par-dessus les barrieres. des disciplines. des fonctions dans l'entreprise, des lieux de travail, des autorités de tutelle, qu'ils n'ont l'occasion de parler entre pairs.

On peut résumer cette évolution, ce nouveau stade de la révolution scientifique et technique, en disant que la science est devenue aujourd hui un facteur indispensable de la production. Elle est entièrement prise dans cette nouvelle fonction, et l'on $n$ 'y verra 
"dégradation", "dérogation", que si l’on n'accepte pas l'idée que l'on ne connaît qu'en transformant.

Il en va de même pour la terminologie: si elle a beaucoup patiné, au point d'être en retard atu moment où la demande sociale s'élève et l'interpelle. c'est sans doute pour avoir ignoré la nouvelle donne dans le jeu entre science, lechnique et production: mais c'est aussi qu elle s'est donné pour tâche de décrire, d'entériner, de prescrire ou de prescrire, mais jamais de transformer, par l'analyse des situations réelles du monde moderne pour en aunéliorer de façon réaliste le fonctionnement.

\section{REFERENCWS}

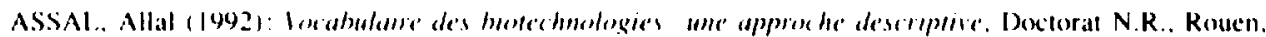
I nuverviti de Rousn. $317 \mathrm{p}$

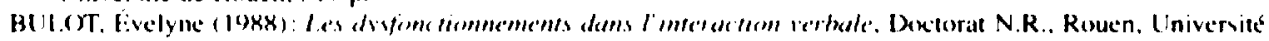
de Rouen. $27 \mathrm{kp}$.

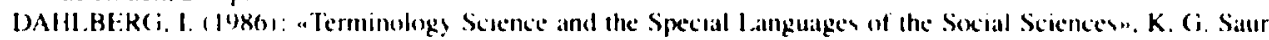

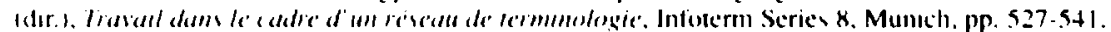

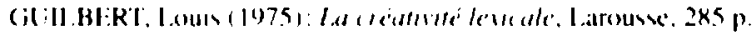

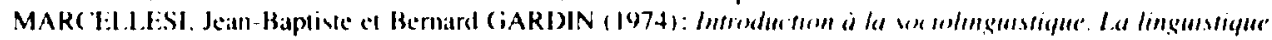
we iale. larousxe. $263 \mathrm{p}$.

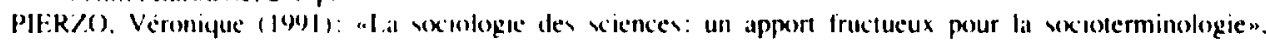

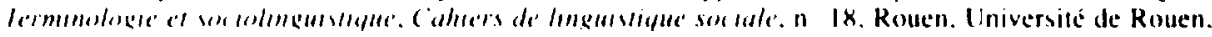
pl. $|x|-2(x)$. 